\title{
TUBERCULOSIS OF BREAST - A STUDY OF 54 CASES IN A TERTIARY LEVEL HOSPITAL IN BANGLADESH
}

\author{
KHAN AS ${ }^{1}$, HAKIM HAN $^{2}$, SULTANA I ${ }^{3}$, TALUKDAR DC ${ }^{4}$, ISLAM MR ${ }^{5}$, HOSSAIN GJ ${ }^{6}$
}

\begin{abstract}
:
Fifty four cases of histopathologically or cytopathologically confirmed tuberculosis of the breast were found during a period of 3 years in surgery outpatient department of Dhaka Medical College Hospital, Dhaka, which was accountable for $2.42 \%$ of all breast diseases. All the patients were female. Most of the cases were in third and fourth decades. Among them, 3 patients were lactating and 1was pregnant. Diagnosis was suspected clinically on the basis of lump or lumpiness and/or solitary or multiple chronic discharging sinuses in the breast or axilla. Breast lumps in 33 cases simulated carcinoma. Histopathological examination was done to establish the nature of the pathology. All patients were treated with four drug combination (4FDC) anti tubercular therapy for 12 months. However, forty patients required some forms of surgery.
\end{abstract}

Key words: breast diseases, tuberculosis of breast, anti-t-*uberculosis therapy.

J Dhaka Med Coll. 2013; 22(2) : 185-187.

\section{Introduction:}

Tuberculosis is a worldwide problem with high incidence in endemic areas such as Indian subcontinent, East Asia and Africa ${ }^{1}$. Tuberculosis of the breast is a rare entity ${ }^{2,3}$. In clinical practice, extrapulmonary manifestations of tuberculosis are commonly seen as meningitis, lymphadenopathy, osteomyelitis, renal and genital tuberculosis. Breast along with spleen and skeletal muscles are considered to be maximally resistant to tubercular infections ${ }^{4-6}$. The overall incidence of tuberculous mastitis is reported to be $0.1 \%$ of all breast lesions, while in developing countries it constituted approximately $3.0 \%$ of surgically treated breast disease ${ }^{7}$. Breast TB is often misdiagnosed as carcinoma breast or pyogenic abscess. The present study represents a clinico-pathological spectrum of tubercular mastitis and stresses the importance of histological examination of breast lumps, so as to avoid unnecessary mastectomies.

\section{Methods:}

Fifty four cases of breast TB were diagnosed after histopathologically or cytopathologically proven during a three year study period (2009-2011) in Surgery Out Patient Department (SOPD) of Dhaka Medical College Hospital, Dhaka. During this period, 2235 patients presented with complaints of breast diseases. Detail history, physical examination and some investigations including cytology or biopsy were done for each patient. This is a retrospective observational study where case numbers and percentages are used. All patients with a breast lump or nodularity were subjected to fine needle aspiration cytology (FNAC). Patients who had a sinus but no lump had undergone excision biopsy of the sinus tract. The cytological findings of epithelioid cell granulomas, Langhans' giant cells, and lymphohistiocytic aggregates confirmed the diagnosis. The discharge from the sinus was subjected to Ziehl-Neelson staining and culture for acid fast bacilli. All patients having palpable axillary lymph nodes were sent for fine needle aspiration cytology (FNAC) as well.

\section{Results:}

During the study period, 2235 patients of breast cases were examined. Among them 54 (2.42\%) patients were found to have been suffering

1. Dr. Arif Salam Khan, Resident Surgeon (General), Department of Surgery, Dhaka Medical College Hospital, Dhaka.

2. Dr. Hafiz Ahmed Nazmul Hakim, Resident Surgeon (Casualty), Department of Surgery, Dhaka Medical College Hospital, Dhaka.

3. Dr. Iffat Sultana, Assistant Professor, Department of Radiology, Bangabandhu Sheikh Mujib Medical University (BSMMU), Dhaka.

4. Dr. Debesh Chandra Talukdar, Assistant Professor, Department of ENT \& Head-Neck Surgery, Dhaka Medical College \& Hospital, Dhaka.

5. Dr. Md.Rafiqul Islam, Assistant Registrar; Department of Neurosurgery, Dhaka Medical College Hospital, Dhaka. 6. Dr. Gazi Jahangir Hossain, Medical Officer, Bhaverchar Union SubCentre, Gajaria, Munshigonj

Correspondence: Dr. Arif Salam Khan, Resident Surgeon (General), Department of Surgery, Dhaka Medical College Hospital, Dhaka. Cell Phone: +8801712044924, Email:khanarifsalam@gmail.com 
from tuberculosis. All the patients were females and their age ranged from 17 to 55 years with $77.77 \%$ of cases $(n=42)$ in age group of $21-40$ years. 3 patients $(6.81 \%)$ were lactating and 1 was pregnant.

Duration of symptoms was between 4 months and 32 months with a mean of 14.4 months. Involvement of right breast was in 23 cases, while left breast 27 cases and bilateral involvement was seen only in 4 cases. Clinical findings are shown in table I. Constitutional symptoms of tuberculosis in the form of fever, weight loss, night sweats, or failing general health were present in only 17 patients $(31.48 \%) .35$ patients $(64.8 \%)$ had a lump in the breast, and $12(22.2 \%)$ of these patients had a discharging sinus in association with the lump (fig 1). Another 9 patients (16.7\%) had multiple sinuses without an underlying lump. 4 patients $(7.4 \%)$ had symptoms of mastalgia and tender nodularity without a cyclical pattern, and $29(53.7 \%)$ had associated axillary lymphadenopathy in the same side. Diagnosis of tuberculosis mastitis was suspected clinically only in $27(50 \%)$ cases. These cases presented with solitary or multiple chronic discharging sinus in the breast with or without underlying lump. Constitutional symptoms of tuberculosis in the form of fever, weight loss, night sweats, or failing general health were present in only 17 patients $(31.48 \%)$. The cytological findings of epithelioid cell granulomas, Langhans' giant cells, and lymphohistiocytic aggregates confirmed the diagnosis of TB. The discharge from the sinus was subjected to Ziehl-Neelson staining and culture and all the specimens proved negative for acid fast bacilli. Among 29 patients having palpable axillary lymph nodes, 23 (79.31\%) patients' lymph nodes were positive for granulomatous inflammation. Rest of them showed reactive changes.

The diagnosis of mammary tuberculosis was confirmed by a combination of clinical suspicion and cytological findings. All patients received antituberculous therapy comprising rifampicin $600 \mathrm{mg}$, isoniazid $300 \mathrm{mg}$, pyrazinamide $1500 \mathrm{mg}$, and ethambutol $800 \mathrm{mg}$ per day (4 FDC) for two months followed by rifampicin and isoniazid for another 10 months. 40 patients $(74.07 \%)$ required surgical intervention. This included excision of mass in 17 patients $(31.48 \%)$, excision of lump and sinus tract in 8 cases $(14.81 \%)$, excision of sinus tract in $9(16.7 \%)$ cases, repeated aspirations from cold abscesses in 6 patients (11.1\%) (table-II).

Table-I

Symptoms of breast TB patients $(n=54)$

\begin{tabular}{lc}
\hline Symptoms & Numbers (\%) \\
\hline Breast lump & $23(42.6)$ \\
Breast lump with sinus & $12(22.2)$ \\
Chronic Sinus & $9(16.7)$ \\
Tender nodularity & $4(7.4)$ \\
Recurrent abscess & $6(11.1)$ \\
Axillary lymphadenopathy & $29(53.7)$ \\
Constitutional symptoms & $17(31.48)$ \\
\hline
\end{tabular}

Table-II

Therapeutic modalities $(n=54)$

\begin{tabular}{lc}
\hline Treatment & No. of patients (\%) \\
\hline Anti Tubercular drugs & $54(100)$ \\
Excision of lumps & $17(31.48)$ \\
Excision of lumps and sinus & $8(14.81)$ \\
Excision of sinus tract & $9(16.7)$ \\
Repeated drainage of abscess & $6(11.1)$ \\
\hline
\end{tabular}

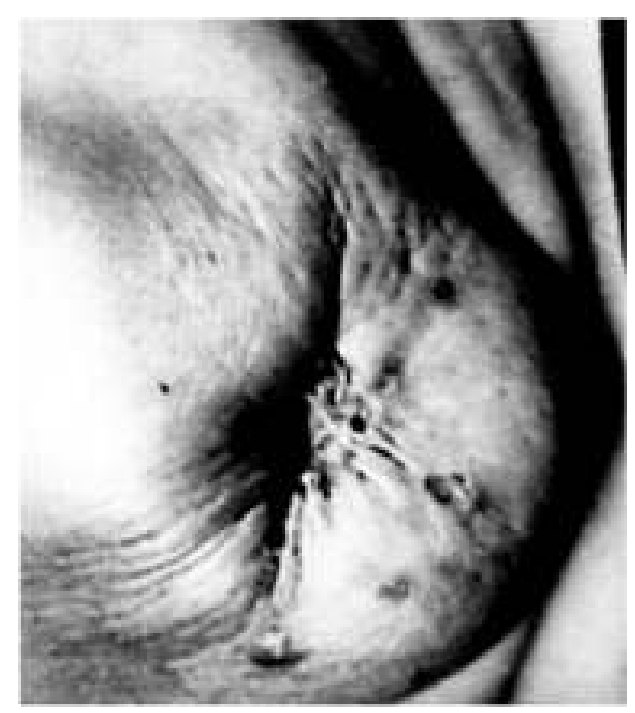

Fig. 1. Multiple sinuses in left breast.

All patients were followed up for 12 months. 14 patients (25.93\%) responded to antituberculous therapy alone while 40 patients required additional surgical treatment as detailed above.

\section{Discussion}

The frequency of tuberculosis of breast among various diseases of breast is high in our 
patients $(2.42 \%)$ as compared to most of the Western series where it is reported to vary from 0.5 to $1.04 \%^{2}$. However, our findings are fairly comparable with those in other Indian studies ${ }^{3}$. There are no definite clinical signs of tuberculosis of the breast. Nevertheless, it should be suspected if there is a lump with chronic discharging sinus, lump in breast with sinus in axilla related to lymphnodes, chronic painful tender lump without any other signs of inflammation and often it mimics carcinoma breast. It is, thus, desirable to wait for histopathological report before undertaking mastectomy. The occurrence of tuberculosis of the breast is common to females of lactating and/or reproductive age group. Presence of milk in lactating breast may make it more susceptible to acute or chronic infection; but involvement in the reproductive age group is not very clearly understood. It needs to be investigated further whether it has any association with hormonal changes occurring in the breast, making the organ more susceptible to infections. There was no male patient in this study though its occurrence in men has been reported 7 . Pathologically, the different forms of breast tuberculosis are (a) acute miliary tuberculosis-manifestation in the breast, as a part of the generalised miliary disease, is extremely rare; (b) nodular tuberculosis which has caseation and abscess formation is most common 8 ; (c) Sclerosing tubercular mastitis, which presents as hard fibrous mass; and (d) duct infection producing marked epithelial fibrosis (tubercular mastitis obliterans). Histologically, diagnostic criteria of breast tuberculosis are presence of caseating granuloma with or without demonstrable acidfast bacilli (AFB) in the section. In the absence of caseation in the breast lump, the caseation in axillary lymph nodes should be taken into consideration but there should be no duct ectasia and plasma cell infiltration in the breast lump 9 . Association of Carcinoma with tuberculosis is extremely rare ${ }^{10}$. Apart from carcinoma, differential diagnosis of breast tuberculosis includes few rare diseases like traumatic fat necrosis, plasma cell mastitis, chronic pyogenic abscess, mammary dysplasia, fibroadenoma, granulomatous mastitis, sarcoidosis or fungal granulomas. Identification of breast tuberculosis does not exclude concomitant breast cancer as both conditions can occasionally co exist ${ }^{11-13}$.
Breast tuberculosis is almost always secondary to a lesion elsewhere in the body. The most common mode of involvement is retrograde lymphatic extension from primary foci in the lungs or mediastinal, parasternal, cervical or axillary lymph nodes ${ }^{14}$. We found that $53.7 \%$ of our cases had associated axillary lymphadenopathy.

\section{Conclusion}

Though tuberculosis of the breast is an uncommon condition, it is a definite clinical entity. It may mimic benign or malignant lesions of breast. No major surgery on the breast should be undertaken without prior histopathological examination. Mastectomy is never indicated in breast tuberculosis and surgery is limited to excision of sinuses and/ or lumps, incision, drainage and curettage of chronic abscesses.

\section{References}

1. Gupta RL. Surgical infections: tuberculosis at other sites-tuberculosis of breast. In: Association of Surgeons of India. Textbook of Surgery. Tata McGraw Hill; 2003: p.130.

2. Kalac N, Ozkan B, Bayiz H, Dursun AB, Demirag F. Breast tuberculosis. Breast 2002; 11: 346-9.

3. Kakkar S, Kapila K, Singh MK, Verma K. Tuberculosis of the breast: a cytomorphologic study. Acta Cytol 2000; 44: 292-6.

4. Kao PT, Tu MY, Tang SH. Tuberculosis of the breast with erythema nodosum : a case report. J Med Case Reports 2010; 4: 124.

5. Murat K, Mehmet T, Sule B, et al. Tuberculosis of breast. Eur J Gen Med 2010; 7: 216-9.

6. Madhusudhan KS, Gamanagatti S. Primary breast tuberculosis masquerading as carcinoma. Singapore Med J 2008; 49: e3.

7. Alagaratnam TT, Ong GB. Tuberculosis of the breast. Br J Surg 1980; 67: 125.

8. Morgen M. Tuberculosis of the breast. Surg Gynae Obstet 1931; 53: 593.

9. Dharkar RS, Kanhere MH, Vaishya ND, Bisarya AK. Tuberculosis of the breast. J Ind Med Assoc 1968; 50: 207.

10. Miller RE, Solomon PP, West JP. Coexistence of carcinoma and tuberculosis of the breast and axillary nodes. Am J Surg 1971; 121: 338.

11. Kao PT, Tu MY, Tang SH. Tuberculosis of the breast with erythema nodosum : a case report. journal of medical case reports 2010;4:124.

12. Tewari M, Shukla HS. Breast tuberculosis: diagnosis, clinical features and management. Indian J Med Res 2005; 122: 103-10.

13. Shinde SR, Chandawarkar RY, Deshmukh SP. Tuberculosis of the breast masquerading as carcinoma: a study of 100 patients. World J Surg 1995; 19: 379-81.

14. Tiwari VS, Agarwal A, Singh PK, Upadhyaya GC. Tuberculosis of the breast. Ind J Tub 1990; 37: 149. 